\title{
JUSTIFICATION OF ACCELERATOR PARAMETERS OF FEEDING HARVESTER UNLOADING CHANNEL
}

Viktor Maksimenko ${ }^{1}$, Volodymyr Kuzmenko ${ }^{1}$, Lyubov Shymko ${ }^{2}$, Vasyl Achkevych ${ }^{2}$

\author{
${ }^{1}$ National Scientific Centre "Institute for Agricultural Engineering and Electrification" of Ukraine, \\ Ukraine; ${ }^{2}$ National University of Life and Environmental Sciences of Ukraine, Ukraine \\ v.v.max@ukr.net,vfkuzmenko@ukr.net,shymkolyuba@ukr.net, achkevychv@gmail.com
}

\begin{abstract}
The article is devoted to increasing the efficiency of forage harvesting equipment based on the substantiation of the parameters of the cut mass accelerator in the unloading channel. The corresponding structural and functional scheme of the cut mass accelerator in the unloading channel of the forage harvester is substantiated in the article. The conditions of the mode of motion of particles of the cut mass in the zone of action of the accelerator without collision with the inner surface of the blade, which can be used in calculating the speed of the working bodies of the accelerator, are theoretically substantiated. A mathematical model of the motion of the selected particle of the cut stem mass has been developed, which connects the main design parameters and conditions of hitting and ascending the particle. This allowed us to set the motion parameters $(\ddot{\tau}, \dot{\tau}, \tau ; \ddot{\epsilon}, \dot{\epsilon}, \epsilon)$ and the angle $\gamma$ between the velocities $\dot{\tau}$ and $\dot{\epsilon}$, and the system of differential equations describes the motion of the cut mass particle from the moment it hits the accelerator blade and climbing from it. As a result of experimental studies, it was found that of the studied structural and kinematic parameters $(v, n, \alpha, \Delta)$, while minimizing the specific energy consumption there is the most significant gap $\Delta$ between the ends of the blades and the accelerator casing, while optimizing the force on the shield-catcher, the speed of the ends of the blades $v$ and the angle of installation of the blade is relative to the radial direction $\alpha$. The dependences of the circumferential velocity of the blade ends $v$ and the gap $\Delta$ between the blades and the casing in both cases are parabolic, and the angle of inclination of the blades $\alpha$ to the radial direction and their number $n$ is linear. The minimum specific energy consumption and the maximum effort on the shield-catcher within the studied parameters are achieved at $v=37$ $40 \mathrm{~m} \cdot \mathrm{s}^{-1}, n=4-6 \mathrm{pcs}, \alpha=0$ to $-5^{\circ}, \Delta=7-9 \mathrm{~mm}$.
\end{abstract}

Keywords: forage harvester, accelerator, cut stemmas, blades, particle motion.

\section{Introduction}

The result of all efforts of the agricultural producer invested in cultivation, preparation and preservation of the future forage depends on the correct choice of the forage harvester and its uninterrupted work. The main characteristic is the correct combination of the productivity of the forage harvester with the consumption of specific energy per unit of feed.

In works [1-3] it is specified that the maximum productivity of the forage harvester makes at the size of the crushed particles from 8 to $19 \mathrm{~mm}$. It is established that most combines over $75 \%$ use a drum shredder. In works [4-6] the energy consumption of the technological process of grinding and transportation of mass is given. Energy consumption is reduced, and the reliability of the device is increased when using bitumen-knife grinders, which perform multi-plane cutting at a speed of 4-8 $\mathrm{m} \cdot \mathrm{s}^{-1}$. The average specific energy required for grinding corn ranged from 2.5 to $5.9 \mathrm{kWh}$ [7].

One of the main components of the forage harvester is the accelerator of the cut mass in the unloading channel. Its main function is to provide acceleration and compactness of the flow of cut mass, after passing the grinding rollers. The use of an accelerator is a must for modern forage harvesters with roller shredders of the biological mass of corn. Grinding of grain increases the digestibility of feed, namely, starch by $64.6 \%$ in the stomach of cattle. Grinding allows to increase the efficiency of silage feeding by $18 \%$. However, this effect is the result of using not only the accelerator, but also the grinder. The cut stem mass after passing of crushing rollers is considerably slowed down that caused the need for installation of the accelerator.

The choice of rational parameters of the accelerator allows to reduce the energy consumption from 0.01 to $0.3 \mathrm{~J} \cdot \mathrm{kg}^{-1}$. The specific heat of combustion of diesel fuel is $43 \mathrm{MJ} \cdot \mathrm{kg}^{-1}$. After the calculation we get a slight reduction in fuel consumption.

Use of the accelerator allows to change the vehicle transporting cut weight to storage without a stop of the forage harvester. This replacement allows to increase the hourly rate of the combine by 1 ton of cut biological mass of corn.

Recent studies of unloading channels in forage harvesters do not fully cover the design parameters and quality of the cutter accelerator. There is a need to justify these parameters. 
In this regard, the justification of the parameters of the cutter accelerator in the unloading channel of the forage harvester is relevant.

\section{Materials and methods}

In the area of action of the accelerator, the particles of mass can interact with its blades in two ways. According to the first variant, the particle moving along a trajectory coinciding with the inner wall of the channel with its speed, catching up with the inner surface of the blade point is slowed down, transferring part of the energy of the rotor (blade) of the accelerator and moves further with the speed of the blade. This part in the area of the accelerator is overtaken by the next rotor blade and the part falls on the outer working side. According to the second option, the outer surface of the blade catches up with the particle, falling on the surface of the blade without bouncing off it, receiving acceleration moves along the surface to the periphery of the blade.

Theoretical preconditions of the process of movement of the crushed cut stem mass are substantiated, which, coming from the knife of the cutting drum in the unloading channel of the forage harvester, is transported due to the received kinetic energy and air flow [8;9].

The formula for the velocity of the particle that does not hit the walls of the unloading channel.

$$
V=\frac{1}{k_{i}}\left[\left(g+k_{i} v_{\mathrm{B}}\right) e^{-k_{i} t}-g\right]
$$

where $k_{i}$-coefficient of proportionality;

$g$ - acceleration of gravity, $\mathrm{m} \cdot \mathrm{s}^{-2}$

$v_{\mathrm{B}}$ - centrifugal velocity of the blade, $\mathrm{m} \cdot \mathrm{s}^{-1}$.

After leaving the rollers, the mass moves along the channel by a flow of particles whose trajectories are parallel to the axis of the channel, or slightly deviate in one direction or another. Once on the walls of the channel, they expend kinetic energy and continue to move along the walls of the channel and fall into the accelerator of the cut stem mass Fig. 1.

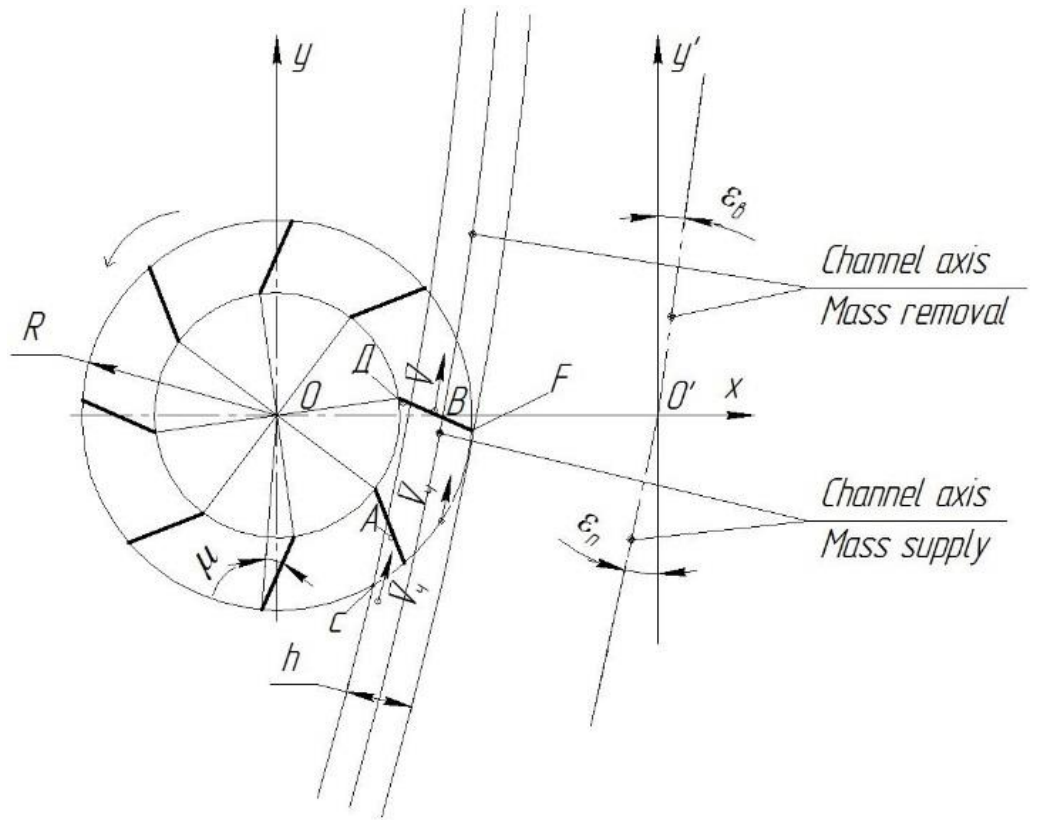

Fig. 1. Interaction of the accelerator with particles of stalk forage

To conduct the research, an experimental setup was developed, the technological scheme of which is presented in Fig. 2. Its main component is a rotor-drum 1 mounted on a frame and closed by a casing. The casing has a receiving neck and an unloading deflector directed to the top. The diameter of the drum rotor is $760 \mathrm{~mm}$, it has 6 blades mounted on the disk. For its drive the electric motor ASIB3-160L$15 \times 1500$ with a power of $15 \mathrm{~kW}$ with a speed of $1460 \mathrm{rpm}$ is used. The electric motor is mounted on the same frame with the rotor-drum. Due to the belt drive, the angular velocity of the rotor-drum 1 is $85.3 \mathrm{~s}^{-1}$ and, accordingly, the circular speed of the ends of the blades is $32.4 \mathrm{~m} \cdot \mathrm{s}^{-1}$. 
The drive of the accelerator 2 is made of an electric motor with power $N_{1}=14 \mathrm{~kW}$ with a speed of $1460 \mathrm{rpm}$ through the variator VC-2 and a belt drive with pulleys $d_{1}=312 \mathrm{~mm}$ and $d_{2}=235 \mathrm{~mm}$. This design of the drive allows to change the speed of the rotor of the accelerator in the range of 54.6$267.5 \mathrm{rad} \cdot \mathrm{s}^{-1}$. A confuser is mounted on the discharge neck of the accelerator, which narrows the flow of mass and directs it to the shield-catcher 4, installed at a distance of $1.05 \mathrm{~m}$ from the confuser. The peculiarity of the shield 4 is that its receiving plane is installed perpendicular to the axis of the confuser - accelerator. Another feature is that the shield-catcher is attached to the rack using a strain gauge. The latter makes it possible to determine the force with which the flow of the cut stem mass interacts with the receiving plane.

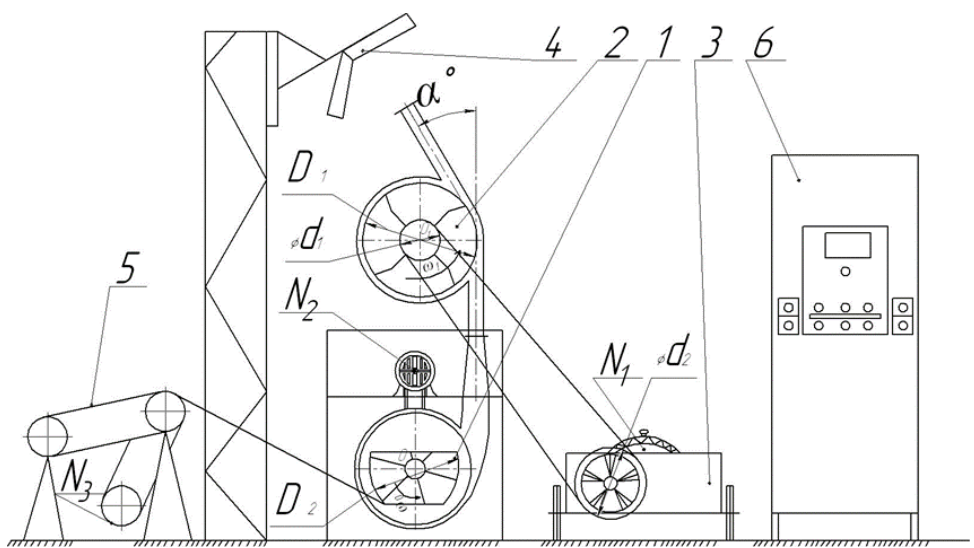

Fig. 2. Scheme of the experimental installation: 1 - drum; 2 - accelerator; 3 - variator; 4 - shield catcher; 5 - conveyor; 6 - control cabinet

In the process of studying the operation of the accelerator, the following indicators are determined: consumed by the drive motor of the accelerator $N_{1}$, the force arising on the receiving plane of the shieldcatcher $F$.

Relative humidity and weighted average cutting length are determined by sampling according to standardized methods. Factors influencing the process of acceleration of the cut stem mass are divided into two groups: technological and structural.

The technological include humidity, cutting length and the amount of mass supply, because they can change, affecting the process and do not depend on the design of the accelerator in Fig. 3. The design parameters characterize the structure of the accelerator and are not interrelated with the technological ones.

The rotor of the accelerator 2 in Fig. 3. is made in such a way that it is possible to install 2, 4, 6 blades in two rows, and the blades can be shifted by half a step of their installation. Adjustment of the angle of inclination of the blades to the radial direction within $+15^{\circ}, 0^{\circ},-15^{\circ}$. Thanks to the variator, the circular velocity of the blade ends was adjusted in the range from 35 to $55 \mathrm{~m} \cdot \mathrm{s}^{-1}$, and the movement of the casing wall relative to the accelerator adjusted the gap between the blade ends and the casing from 2 to $10 \mathrm{~mm}$.

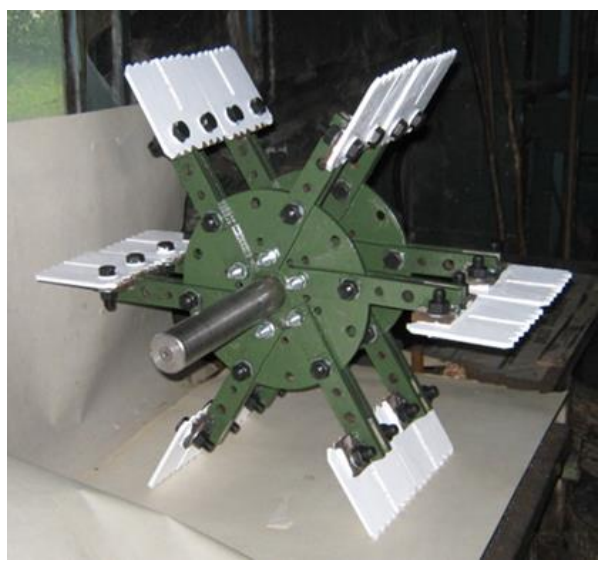

Fig. 3. Laboratory sample of the accelerator of the cut stem mass 
Power was measured by recording the current using a suitable laboratory transformer UTT-6M2, accuracy class 0.2 . Before the experiments, the laboratory transformer was calibrated using a measuring complex K505. A PC (personal computer) with an E14-140-M-D analog-to-digital converter and appropriate software was used to record the signal from the transformer.

Since theoretical studies allow us to determine only the kinematic parameters of the motion of the particle as a result of the action of the accelerator, the determination of the energy performance of the acceleration process was determined experimentally.

Studies of the influence of technological factors on the specific energy consumption and the force of interaction of the cut mass with the shield-catcher were performed as one-factor experiments.

The influence of the accelerator parameters was determined using a three-level plan of a four-factor experiment.

\section{Results and discussion}

As a result of processing the obtained data, the regression equation of specific energy consumption in decoded form is obtained:

$$
\begin{aligned}
E_{n}= & 0.0388299+0.0311599 n-0.0390452 \Delta+0.0000563 v^{2}++0.0001392 v \alpha- \\
& -0.00011392 n \alpha+0.0024968 \Delta^{2}
\end{aligned}
$$

where $v$-angular velocity of the ends of the blades, $\mathrm{m} \cdot \mathrm{s}^{-1}$;

$\alpha$ - angle of the blades to the radial direction, ${ }^{\circ}$;

$n$ - number of blades, pcs;

$\Delta$ - gap between the casing and the ends of the blades, $\mathrm{mm}$.

For dependences (2), which are adequate at $95 \%$ confidence level, the coefficient of multiple determination is $D=0.95$. The value of the Fisher test $F=53.93$, which is greater than the tabular values. All model coefficients are significant at a confidence level of at least $95 \%$.

For a clear analysis of the influence of the parameters on the specific energy consumption, the dependences on the parameters of the regression equation subtracted to the square $(\Delta)$ and influencing linearly $(\alpha)$ are constructed.

The range of variation of the gap between the end of the blade and the casing of the accelerator of the cut mass is $2-10 \mathrm{~mm}$. Increasing the gap to $7 \mathrm{~mm}$ reduces the specific energy consumption. Reaching a minimum at a gap of 7-9 $\mathrm{mm}$, its further increase leads to an increase in specific energy consumption. Thus, in the research area there is an extremum in the gap area of 7-9 $\mathrm{mm}$.

The dependence of energy consumption on the angle of deviation of the blade in the radial direction and their number at a gap of $6 \mathrm{~mm}$ and the velocities of the ends of the blades $35,45,55 \mathrm{~m} \cdot \mathrm{s}^{-1}$ is presented in Fig. 4.

Increasing the angle of deviation from $-15^{\circ}$ to $+15^{\circ}$ in the radial direction leads to a uniform increase in the specific energy consumption for mass acceleration in Fig. 5. Therefore, at $\alpha=-15^{\circ}$, the minimum consumption of specific energy by the accelerator within the change of the studied parameters reaches, $E_{n}=0.03-0.08 \mathrm{~J} \cdot \mathrm{kg}^{-1}$.

At the maximum values of the analysed parameters $\alpha=15^{\circ}$ specific energy consumption reaches the maximum value of $E_{n}=0.15-0.30 \mathrm{~J} \cdot \mathrm{kg}^{-1}$, which is 3.8-5.0 times more than the minimum values.

As a result of experimental studies, it was found that of the studied design parameters $(v, n, \alpha, \Delta)$ the most important is the gap $\Delta$ between the ends of the blades and the casing. The effect of the gap $\Delta$ on the specific energy consumption is parabolic, and the angle of inclination of the blades to the radial direction $\alpha$ is linear. The minimum specific energy consumption within the studied parameters is achieved at $v=35 \mathrm{~m} \cdot \mathrm{s}^{-1}, n=2 \mathrm{pcs}, \alpha=-15^{\circ}, \Delta=7-9 \mathrm{~mm}$.

The force on the shield-catcher characterizes the rate of mass ejection and is a qualitative indicator of the acceleration process. form:

After substituting relations, we obtain the force regression equation on the shield-catcher in decoded 


$$
\begin{aligned}
F_{\mathrm{sch}}= & 352.89-15.0837 v+14.5071 n+0.169083 v^{2}-1.08917 n^{2}-0.27494 n \alpha- \\
& -0.146103 \Delta^{2},
\end{aligned}
$$

$E_{n}, \mathrm{~J} \cdot \mathrm{kg}^{-1}$

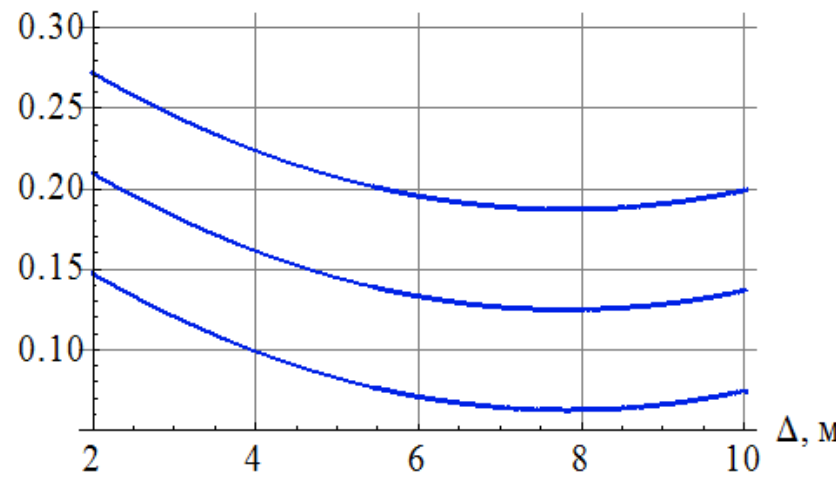

Fig. 4. Influence of the gap $\Delta, \mathbf{m m}$ on the specific energy consumption $E p, \mathrm{~J} \cdot \mathrm{kg}^{-1}$ for the values of

the number of blades n-2, 4 and 6 pes

(respectively, lower, middle and upper surfaces)
$E_{n}, \mathrm{~J} \cdot \mathrm{kg}^{-1}$

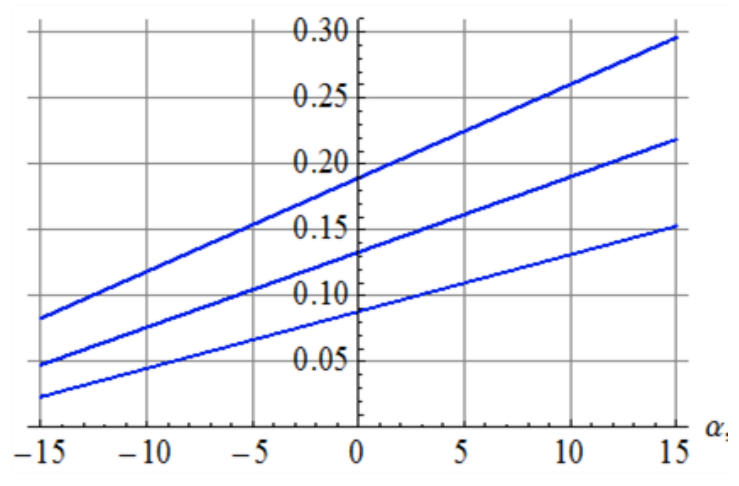

Fig. 5. Influence of the angle of inclination of the blades $\alpha$ on the consumption of specific energy $E n$ for the values of the circular velocity of ends of the blades v-35, 45 and $55 \mathrm{~m} \cdot \mathrm{s}^{-1}$ (lower, middle and upper surfaces, respectively)

For dependences which are adequate at $95 \%$ confidence level, the coefficient of multiple determination is $D=0.95$. The value of the Fisher criterion $F=54.71$, which is greater than the tabular values. All model coefficients are significant at a confidence level of at least $95 \%$.

Graphs are constructed for the visual analysis of influence of the parameters on efforts on a boardcatcher, Fig. 6-7.

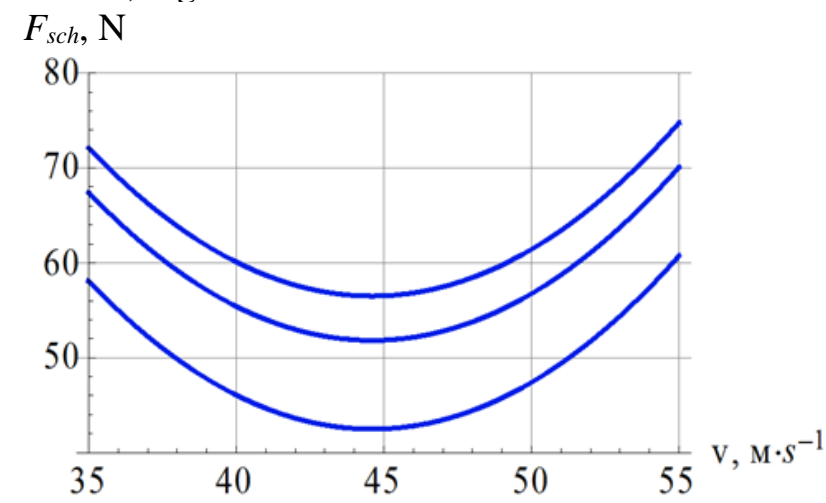

Fig. 6. Influence of circular velocity $\mathbf{v}, \mathbf{m} \cdot \mathrm{s}^{-1}$ on the force on the shield-catcher $F_{s c h}, \mathrm{H}$ for values of the number of blades n-2, 4 and 6 pcs (lower, middle and upper surfaces, respectively)

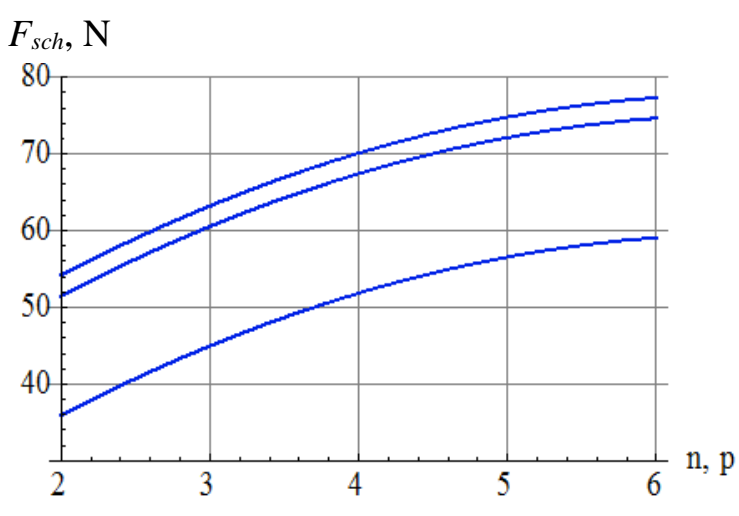

Fig. 7. Influence of the number of blades $n$ on the force on the shield-catcher $F_{c s h}$ for values of the circular velocity of ends of the blades $\mathrm{v}-35,45$ and $55 \mathrm{~m} \cdot \mathrm{s}^{-1}$ (respectively lower, middle and upper surface)

Analysing the obtained graphs, we see that the effect of speed has a minimum in the study area, which is achieved at a speed of the ends of the blades of the accelerator $44-46 \mathrm{~m} \cdot \mathrm{s}^{-1}$. This is explained by the fact that in addition to the organization of the movement of particles of the cut stem mass, there is an interaction of air flows, which is created with the acceleration of the mass.

With increasing number of blades (Fig. 7) from 2 to 6 pieces the force on the shield-catcher created by the flow of the cut stem mass increases along the parabolic curve.

Therefore, at $n=2 \mathrm{pcs}$, the value of the force on the shield-catcher reaches a minimum and within the change of the studied parameters it reaches, $F_{\text {sch }}=35-37 \mathrm{~N}$. 
At the maximum values of the analysed parameters $n=6 \mathrm{pcs}$, the force on the shield-catcher reaches the maximum value $F_{\text {sch }}=78-88 \mathrm{~N}$, which is $2.2-2.4$ times more than the minimum values.

When choosing the parameters of the accelerator we should focus on the minimum energy consumption and maximum effort created on the shield-catcher.

As a result of experimental studies, it was found that of the studied design parameters $(v, n, \alpha, \Delta)$ in minimizing the specific energy consumption is the most important gap $\Delta$ between the ends of the blades and the accelerator casing, in optimizing the force on the shield-catcher, the speed of the blade ends $v$ and the angle of installation of the blade is relative to the radial direction $\alpha$. The dependence of the circular velocity of the ends of the blades $v$ and the gap $\Delta$ between the blades and the casing in both cases are parabolic, and the angle of inclination of the blades $\alpha$ to the radial direction and their number $n$ is linear.

The minimum specific energy consumption and the maximum effort on the shield-catcher within the studied parameters are achieved at $v=37-40 \mathrm{~m} \cdot \mathrm{s}^{-1}, n=4-6 \mathrm{pcs}, \alpha=0$ to $-5^{\circ}, \Delta=7-9 \mathrm{~mm}$.

\section{Conclusions}

1. The conditions of the mode of motion of particles of the cut mass in the zone of action of the accelerator without collision with the inner surface of the blade, which can be used in calculating the speed of the working bodies of the accelerator, are theoretically substantiated.

2. The developed mathematical model of motion of the selected particle of the cut stem mass, which connects the main structural parameters and conditions of impact on the blade and the ascent of the particle, allowed to establish the motion parameters $(\ddot{\tau}, \dot{\tau}, \tau ; \ddot{\epsilon}, \dot{\epsilon}, \epsilon) \dot{\tau}$ i $\dot{\epsilon}$, and the angle $\gamma$ between the velocities $\dot{\tau}$ and $\dot{\epsilon}$, and the system of differential equations describes the motion of the cut particle of mass from the moment it hits the accelerator blade and its ascent from it.

3. The developed laboratory installation allows to change the frequency of rotation of the accelerator of the cut mass, the gap between the rear wall and the end of the blade, the angle of installation of the blade and the number of blades, which ensures implementation of the experimental research program.

4. As a result of experimental studies, it was found that of the studied structural and kinematic parameters $(v, n, \alpha, \Delta)$ while minimizing the specific energy consumption is the most significant gap $\Delta$ between the ends of the blades and the accelerator casing, while optimizing the force on the shield-catcher, the speed of the ends of the blades $v$ and the angle of installation of the blade is relative to the radial direction $\alpha$. The dependences of the velocity of the blade ends $v$ and the gap $\Delta$ between the blades and the casing in both cases are parabolic, and the angle of inclination of the blades $\alpha$ to the radial direction and their number $n$ is linear.

5. The minimum specific energy consumption and the maximum effort on the shield-catcher within the studied parameters are achieved at $v=37-40 \mathrm{~m} \cdot \mathrm{s}^{-1}, n=4-6 \mathrm{pcs}, \alpha=0$ to $-5^{\circ}, \Delta=7-9 \mathrm{~mm}$.

\section{References}

[1] Li Y., Tao C., Zhe Q., Kehong L. Development and application of mechanized maize harvesters. Int J Agric \& Biol Eng., vol. 9, No.3, 2016, pp. 15-28.

[2] Belov M. Precision cut forage harvester chopper units and particle length distribution. Agric. Eng. Int., vol. 21, No. 4, 2019, pp. 83-89.

[3] Golovkov A., Boiko A., Justification of the choice of the type of forage harvester for farms. Web of Conferences 126, 00053 (2019).

[4] Nedelcu A., Popa D., Ciupercă R., Popa L.. Experimental research with forage harvester for ensilage. International Symposium, ISB-INMA-TEH, Agricultural and Mechanical Engineering, Bucharest, Romania, 31 October-1 November 2019. 2019 pp.356-361.

[5] Kholodiuk O. Design features of shredding machines for feeding machines. Slovak international scientific journal, vol. 3, No. 45, 2020, pp. 20-31.

[6] Niemann J., Dehmer J., Batos A., Kiel J., Seifert S. The impact of electrification to the total cost of ownership of mobile production machines on the example of a forage harvester. 25th Southern African Universities Power Engineering Conference SAUPEC 2017At: Stellenbosch, South Africa.

[7] Zhang M., Sword M. L. Design and evaluation of a corn silage harvester using shredding and flail cutting, American Society of Agricultural Engineers, vol. 46(6), 2003, pp. 1503-1511. 
[8] Kuzmenko V., Maksimenko V., Yampolsky S. Experimental determination of the influence of the design parameters of the mass accelerator of the forage harvester on the specific energy consumption. Mechanization and electrification of agriculture: [national collection]. Vol. 5 (104), Glevaha 2017, pp. 224-231.

[9] Kuzmenko V., Maksimenko V., Yampolsky S. Experimental substantiation of the parameters of the mass accelerator of the forage harvester. Collection of scientific works of the Scientific and Practical Center of the National Academy of Sciences of Belarus for Agricultural Mechanization. Mechanization and electrification of agriculture. Issue No.51, Minsk, 2017, pp. 194-199. 